\title{
Active vibration suppression of a flexible link manipulator using a piezoelectric actuator
}

\author{
H. Salmasi, R. Fotouhi \& P. N. Nikiforuk \\ Mechanical Engineering Department, University of Saskatchewan, \\ Saskatoon, Canada
}

\begin{abstract}
The active vibration suppression of a single flexible link manipulator using a piezoelectric actuator is investigated. For this purpose, a finite element (FE) model is developed for the modal and transient analysis of a cantilever beam and a flexible link manipulator. A proportional control strategy is employed in a FE model to adjust the voltage applied to the piezoelectric actuator so as to control vibration. For the flexible link manipulator, it is shown that the vibration is well suppressed during and at the end of a manoeuvre by locating the piezoelectric actuator at the optimum location. The effect of the value of the controller gain on the vibration behaviour of the system is investigated and the optimum controller gain is found using two main evaluation criteria; these are contribution of dominant frequencies in response and the norms of vibration amplitudes.

Keywords: vibration suppression, cantilever beam, flexible link manipulator, piezoelectric actuator.
\end{abstract}

\section{Introduction}

Designing and utilizing robot manipulators having higher load capacities is always desired. However, vibration is an important factor that restricts the performance of such devices especially in applications where accurate positioning is very important. In the past decade different approaches have been used for vibration suppression. Active vibration control is one of the best approaches to suppress vibration.

One of the methods of active control is using piezeoelectrics as actuators [1]. It is worth noting that piezoelectric is the coupling of structural and electric 
fields. When the piezoelectric used as an actuator, applying a controlled voltage to a piezoelectric changes its shape and a bending force (actuating force) is created due to the piezoelectric deformation.

Piezoelectrics actuators have been successfully used for vibration suppression [2]. Chen and Shen [3] employed the finite element method to model the dynamics of a plate with piezoelectric actuator patches in which it was assumed that each mode could be controlled separately [3]. The optimum placement of the actuators for a cantilevered plate was proposed in [4]. The effect of the placement and the length of the piezoelectric actuator on the modal and spatial controllability of structure was analysed in [5]. The vibration control of cantilever beams was experimentally studied in [6].

In this paper, the active vibration suppression for a cantilever flexible beam and a single-link flexible manipulator using a piezoelectric actuator is described. In Section 2, the mathematical formulation of the piezoelectric actuator is presented. The natural frequencies of the cantilever beam and rotating robot manipulator are obtained. The results of the numerical simulation are reported in Section 3 and the conclusions in Section 4.

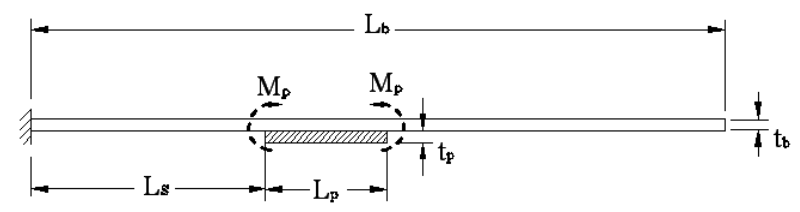

Figure 1: Model of a cantilever beam with piezoelectric actuator.

\section{Mathematical formulation}

\subsection{Piezoelectric actuator}

A cantilever beam with a piezoelectric actuator, shown in Figure 1, was used in the study described in this paper. For perfectly bonded piezoelectric actuators and assuming an Euler-Bernoulli beam, the moment induced by the applied voltage on the piezoelectric actuator is given as [8]

$$
M_{p}=E_{p} e_{31}\left[\left(V_{1}-V_{2}\right)\left(t_{b} / 2+t_{p} / 2\right)\right]
$$

where $E_{p}$ is the module of elasticity of the piezoelectric element, $e_{31}$ the piezoelectric actuator constant, $t_{b}$ the thickness of the beam and $t_{p}$ the thickness of the piezoelectric actuator, respectively. $V_{1}$ and $V_{2}$ are respectively the applied voltage to the top and bottom surfaces of the piezoelectric actuator, and $M_{p}$ is the effective bending moment applied to the beam with an equivalent area moment of inertia $I_{e q}$ where 


$$
I_{e q}=(1 / 12) A_{b} t_{b}^{2}+(1 / 12) A_{p} t_{p}{ }^{2}+\left[\frac{(1 / 2)\left(t_{b}+t_{p}\right)}{1-r_{A} r_{E}}\right]^{2}\left[A_{b}+A_{p}\left(r_{E} r_{A}\right)^{2}\right]
$$

In this equation, $A_{b}$ and $A_{p}$ are the areas of the beam and piezoelectric actuator, respectively, and $r_{E}$ and $r_{A}$ are the ratios of the module elasticity and area of the beam to those of the piezoelectric actuator, respectively. By letting $K_{s}=(1 / 2) E_{p} e_{31}\left(t_{b}+t_{p}\right)$, eqn (1) becomes

$$
M_{p}=K_{s}\left(V_{1}-V_{2}\right)
$$

If the applied voltage to the bottom surface of the piezoelectric actuator is zero $\left(V_{2}=0\right)$, then from eqn (3) $M_{p}$ will be proportional to the applied voltage on the top surface, $M_{p}=K_{s} V_{1}$. If the beam is modelled as a Euler-Bernoulli beam with deflection $y(x, t)$, where the $x$ is measured from the fixed end of the beam and $t$ is time, the partial differential equations of the system becomes

$$
E_{b} I_{e q} \frac{\partial^{4} y(x, t)}{\partial x^{4}}+\rho_{b} \frac{\partial^{2} y(x, t)}{\partial t^{2}}=K_{s} V_{1} \frac{\partial}{\partial x}\left[\delta\left(x-L_{s}-L_{p}\right)-\delta\left(x-L_{s}\right)\right]
$$

where $E_{b}$ and $\rho_{b}$ are the module of elasticity and density of the beam respectively. $L_{b}, L_{p}$ and $L_{s}$, as shown in Figure 1, are the length of the beam, length of the piezoelectric actuator, and distance of the piezoelectric actuator from the fixed end respectively, and $\delta(x)$ is the Dirac function.

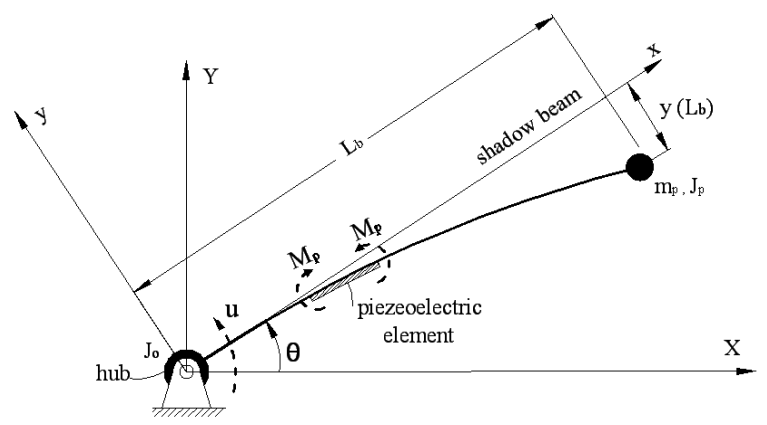

Figure 2: A single flexible link robot manipulator including its tip mass and hub inertia.

\subsection{Dynamics of manipulator}

The manipulator shown in Figure 2 has a hub at the base with mass moment of inertia $J_{o}$, a beam of length $L_{b}$, a payload with mass $m_{p}$ and mass moment of inertia $J_{p}$. The coordinate system $(X, Y)$ is fixed at the hub and the coordinate system $(x, y)$ rotates with angular velocity $\dot{\theta}$ where the angle $\theta$ is the rotation of the base. Torque $u$ applies to the hub and the arm rotates around its base 
during the interval time between zero and $t_{d}$ (manipulator reaches its desired destination). After reaching the desired angle $\theta_{d}$, the torque reduces to zero and the arm behaves as a cantilever beam for $t>t_{d}$. Thus, the simulation procedure must be performed in two steps; first for a rotating flexible manipulator for $0<t \leq t_{d}$, and then for a cantilever beam for $t>t_{d}$. Piezoelectric actuator is used for vibration suppression of the manipulator during the rotation of the arm and after the arm is reached its desired position. The equations of motion of the rotating flexible manipulator can be divided into two differential equations. The first equation is related to the flexible behaviour of the manipulator and second describes the hub's rigid body motion [9]

$$
\begin{aligned}
& E_{b} I_{e q}\left(\partial^{4} / \partial x^{4}\right) y(x, t)+\rho_{b}(\ddot{y}(x, t)+x \ddot{\theta}(t))=M_{p} \frac{\partial}{\partial x}\left[\delta\left(x-L_{s}-L_{p}\right)-\delta\left(x-L_{s}\right)\right] \\
& I \ddot{\theta}(t)+\mu(t)=u
\end{aligned}
$$

where the dot indicates the time derivative and ()$^{\prime}$ represents the derivation with respect to $x$ and

$$
\begin{aligned}
& \mu(t)=\rho_{b}\left[\int_{o}^{L_{b}} x \ddot{y}(x, t) d x\right]+m_{p} L_{b} \ddot{y}\left(L_{b}, t\right)+J_{p} \ddot{y}^{\prime}\left(L_{b}, t\right) \\
& I=J_{o}+J_{p}+(1 / 3) \rho_{b} A_{b} L_{b}{ }^{3}+m_{p} L_{b}{ }^{2}
\end{aligned}
$$

The boundary conditions for eqn (5) are

$$
\begin{aligned}
& y(t, 0)=y^{\prime}(t, 0)=0, E_{b} I_{e q}\left(\partial^{2} / \partial x^{2}\right) y\left(L_{b}, t\right)=-J_{p}\left[\ddot{\theta}(t)+\ddot{y}^{\prime}\left(L_{b}, t\right)\right] \\
& E_{b} I_{e q}\left(\partial^{3} / \partial x^{3}\right) y\left(L_{b}, t\right)=m_{p}\left[L_{b} \ddot{\theta}(t)+\ddot{y}\left(L_{b}, t\right)\right]
\end{aligned}
$$

where the first two boundary conditions are due to the fact that the manipulator is clamped at the base, and the displacement and the slope of the beam at base must be zero. The other two boundary conditions represent the balance of the moment and force at the tip of the manipulator.

The characteristic equation can be derived from eqn (5) and eqn (7) by setting external torques to zero i.e. $u=0$ and $M_{p}=0$. Thus

$$
\begin{aligned}
& c \cdot s h-s \cdot c h-\left(2 m_{p} / \rho_{b}\right) \beta_{i} s \cdot s h-\left(2 J_{p} / \rho_{b}\right) \beta_{i}^{3} c \cdot c h-\left(J_{p} / \rho_{b}\right) \beta_{i}{ }^{3}(1+c \cdot c h)- \\
& \left(m_{p} / \rho_{b}{ }^{2}\right) \beta_{i}^{4}\left(J_{o}+J_{p}\right)(c \cdot s h-s \cdot c h)+\left(J_{o} J_{p} / \rho_{b}{ }^{2}\right) \beta_{i}{ }^{6}(c \cdot s h+s \cdot c h)- \\
& \left(J_{o} J_{p} m_{p} / \rho_{b}{ }^{3}\right) \beta_{i}{ }^{7}(1-c \cdot c h)=0
\end{aligned}
$$

where

$\omega_{i}=\beta_{i}{ }^{2} \sqrt{\left(E_{b} I_{e q} / \rho_{b} A_{b}\right)}, s=\sin \left(\beta_{i} L_{b}\right), c=\cos \left(\beta_{i} L_{b}\right), s h=\sinh \left(\beta_{i} L_{b}\right), c h=\cosh \left(\beta_{i} L_{b}\right)$ The manipulator behaves as a cantilever beam after reaching the desired position, and the angular acceleration $\ddot{\theta}$, becomes zero. The characteristic equation for a cantilever beam [10] is then reduced to

$$
\begin{aligned}
& (1+c \cdot c h)-\left(m_{p} / m_{b}\right) \beta_{i}(s \cdot c h-c \cdot s h)-\left(J_{p} / 3 J_{o}\right) \beta_{i}^{3}(s \cdot c h+c \cdot s h)- \\
& \left(m_{p} J_{p} / 3 m_{b} J_{o}\right) \beta_{i}^{4}(1-c \cdot c h)=0
\end{aligned}
$$


The natural frequencies of the system can be now computed using characteristic eqns (8) and (9) during manoeuvre of the rotating manipulator $\left(0<t \leq t_{d}\right)$ and after reaching the desired rotation $\left(t>t_{d}\right)$.

\subsection{Controller}

A proportional controller was used in which the applied voltage to the piezoelectric actuator was proportional to the axial strain. A block diagram of the controller is shown in Figure 3 where $K_{c}, \varepsilon, V$ and $V_{\varepsilon}$ are the gain, strain at the midpoint of the piezoelectric actuator, potentiometer output voltage and applied voltage to the piezoelectric actuator, respectively. To avoid the instability due to the non-collocation of sensor and actuators [11], the actuator and sensor were located at the same location; that is the strain $\varepsilon$ was measured at the location of the piezoelectric actuator. The set point (for error) was selected as zero.

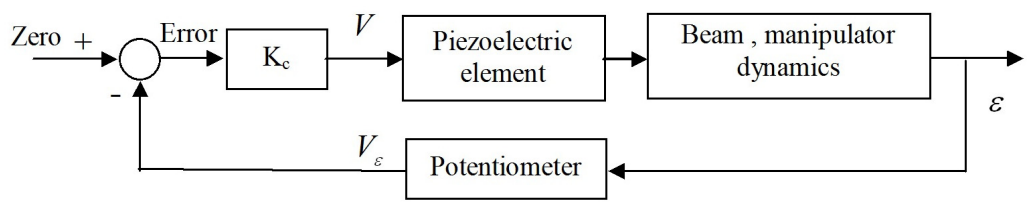

Figure 3: Block diagram of the vibration control system.

\section{Simulation results}

Three types of elements from the ANSYS [12] elements library were used to model the beam/manipulator. The beam was constructed using 10 "PLANE 82" elements spaced equally along beam. Since "PLANE 82" did not have a rotational degree of freedom, two "BEAM 3" elements having three degrees of freedom (translations in the local $\mathrm{x}$ and $\mathrm{y}$ directions as well as rotation in the $\mathrm{z}$ direction) were used for the base rotation. The element "PLANE 223", which models the piezoelectric actuator, was used to suppress the vibration.

Sampling time is an important parameter in numerical simulation. Smaller sampling time gives more accurate results, but increases the simulation time. Thus, a compromise between the accuracy and the simulation time is necessary. For an efficient and accurate response, the time step $(\Delta t)$ is usually set to capture 20 points per cycle for a signal with the frequency of $f_{d}$; that is $\Delta t=1 / 20 f_{d}$, where $f_{d}$ is the highest desired frequency to capture [13]. In this simulation, the third natural frequency was selected as the desired highest frequency. In the simulation study both modal and transient analyses were carried out for the cantilever beam and for the robot flexible link manipulator. The simulation was performed in two steps. In the first step, the effect of placement of the piezoelectric actuator on the vibration was studied and the 
optimal location of the piezoelectric actuator along the beam was determined. In the next step, the active vibration suppression of the robot flexible link manipulator during and at the end the manoeuvre was successfully accomplished, and the effect of the controller gain on the vibration behaviour of the system was determined.

\subsection{Optimum location of the piezoelectric actuator}

Since the location of the piezoelectric actuator has a significant effect on the control of the vibration [4,7], different locations of the piezoelectric actuator along the beam, i.e., $L_{s} / L_{b}=0.05,0.1,0.2,0.3,0.4,0.5$, were investigated. The beam and piezoelectric actuator shown in Figures 1 and 2, have the following physical properties

$$
\begin{aligned}
& E_{b}=201 \mathrm{e} 9 \mathrm{~N} / \mathrm{m}^{2}, \rho_{b}=7.80 \mathrm{e} 3 \mathrm{~kg} / \mathrm{m}^{3}, L_{b}=0.200 \mathrm{~m}, \\
& L_{s}=0.030 \mathrm{~m} t_{b}=0.001 \mathrm{~m}, v=0.300, I_{b}=8.33 \mathrm{e}-11 \mathrm{~m}^{4}, \\
& L_{p}=0.010 \mathrm{~m}, t_{p}=0.001 \mathrm{~m} \\
& m_{p}=0 \mathrm{~kg}, J_{p}=0 \mathrm{~kg} \cdot \mathrm{m}^{2}, J_{o}=0 \mathrm{~kg} \cdot \mathrm{m}^{2}
\end{aligned}
$$

To find the best location of the actuator, the following evaluation criteria were introduced

$$
E_{2-3}=\sqrt{\frac{1}{N_{3}-N_{2}} \sum_{i=N_{2}}^{i=N_{3}}\left(\frac{y_{i, e}}{y_{\text {max }, e}}\right)^{2}}, E_{0-3}=\sqrt{\frac{1}{N_{3}-1} \sum_{i=0}^{t=N_{3}}\left(\frac{y_{i, e}}{y_{\text {max }, e}}\right)^{2}}
$$

where the norms $E_{0-3}$ and $E_{2-3}$ represent the values of the normalized tip deflections for time intervals of $(0 \leq t \leq 3)$ and $(2 \leq t \leq 3)$, respectively. The $y_{i, e}$ is tip deflection of the beam at the time step $i, N_{3}$ is the number of time steps at $t=3$ and $N_{2}$ is the number of time steps at $t=2$. Table 1 illustrates the values of these norms for the different cases investigated. The values of $E_{0-3}$ and $E_{2-3}$ are plotted versus $L_{s} / L_{b}$ in Figure 4. According to this figure, $L_{s} / L_{b}=0.3$ corresponds to the best location of the piezoelectric actuator. This finding is also consistent with the findings of [4] for a similar beam.

\subsection{Flexible link robot manipulator}

To verify the effectiveness of the piezoelectric actuator in suppressing the vibration of a robot manipulator, a single flexible manipulator was analyzed. In this case, the manipulator could rotate about its base. The physical properties and the dimensions were same as those reported in the previous section, except the values of the mass moment of inertia of the hub and the tip mass which were selected as $J_{o}=1.2 e-3 \mathrm{~kg} \cdot \mathrm{m}^{2}$ and $m_{p}=0.2 \mathrm{~kg}$ respectively. The torque applied to the hub was of a bang-bang nature, as shown in Figure 5(a), causing the manipulator initially to accelerate, then decelerate and finally to lock at its desired final position, when it continued to vibrate as a cantilever beam. As 
shown in Figure 5(b), the hub rotated approximately $0.8 \mathrm{rad}$ in one second and it was locked then at $0.8 \mathrm{rad}$. The natural frequencies of the manipulator and cantilever beam theoretically were obtained using eqns (8) and (9). These natural frequencies are compared in Table 2 against the FEA results.

Table 1: Evaluation criteria for different locations of piezoelectric actuator for a cantilever beam for initial condition $y\left(L_{b}, 0\right)=0.01 \mathrm{~m}$ and controller gain $K_{c}=4.00 e 6$.

\begin{tabular}{|c|c|c|c|c|c|c|}
\hline$L_{s} / L_{b}$ & 0.05 & 0.1 & 0.2 & $\mathbf{0 . 3}$ & 0.4 & 0.5 \\
\hline$E_{0-3}$ & 0.3917 & 0.2999 & 0.2848 & $\mathbf{0 . 2 8 4 5}$ & 0.2938 & 0.3106 \\
\hline$E_{2-3}$ & 0.2084 & 0.0799 & 0.0630 & $\mathbf{0 . 0 6 3 0}$ & 0.0731 & 0.0925 \\
\hline
\end{tabular}

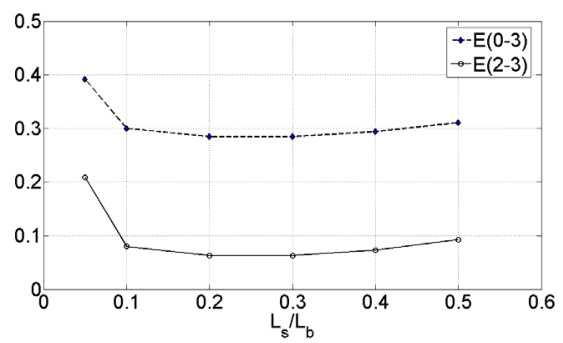

Figure 4: Norms of normalized tip deflection of cantilever beam for optimum location of piezoelectric actuator.

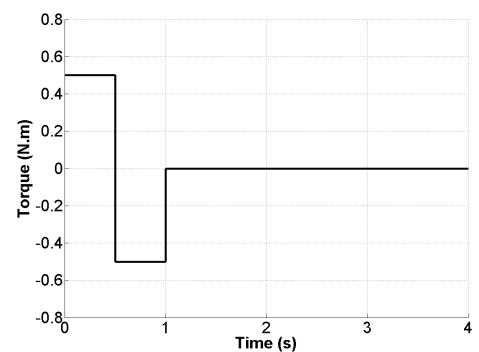

(a)

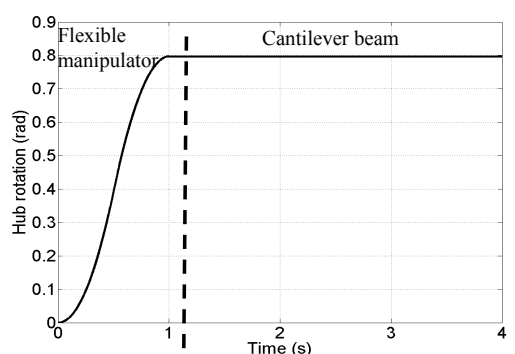

(b)

Figure 5: (a) Applied bang-bang controller torque for flexible link manipulator, and (b) hub rotation.

Figure 6(a) illustrates the tip deflection with respect to the shadow beam (see Figure 2) without the controller being active $\left(K_{c}=0\right)$. To find the dominant frequencies of the system, the FFT of the tip deflections in time was evaluated. This is illustrated in Figure 6(b) which indicates three dominant frequencies. The first was the main excitation frequency which was $1.0 \mathrm{~Hz}$. The second frequency was approximately $17 \mathrm{~Hz}$ which corresponded to the first natural frequency of 
the cantilever beam and the third frequency, which was approximately $60 \mathrm{~Hz}$, corresponded to the first non-zero natural frequency of the flexible link manipulator. To suppress the vibration a piezoelectric actuator was placed at the optimum location, $L_{s} / L_{b}=0.3$, as reported in Section 3.1 for the cantilever beam. Our investigations showed similar behaviour for single flexible link manipulator as well.

The simulation was carried out for different values of the controller gain, $K_{c}=2.00 e 5,4.00 e 5$ and $6.00 e 5$, and these are referred to as Cases 2, 3 and 4, respectively. As shown in Figure 7(a), the vibration was well suppressed during and at the end of the manoeuvre for Case $3\left(K_{c}=4.00 e 5\right)$. The FFT of the tip deflection in time is shown in Figure 7(b) for this case.

Table 2: The first three natural frequencies of the flexible link manipulator and the corresponding cantilever beam.

\begin{tabular}{|c|c|c|c|c|}
\cline { 2 - 5 } \multicolumn{1}{c|}{} & \multicolumn{2}{c|}{ Flexible link manipulator } & \multicolumn{2}{c|}{ Cantilever beam } \\
\hline Set & Theoretical (Hz) & FEA (Hz) & Theoretical (Hz) & FEA (Hz) \\
\cline { 2 - 5 } 1 & 0 & 0 & 16.97 & 16.91 \\
\hline 2 & 61.94 & 61.41 & 112.6 & 110.5 \\
\hline 3 & 145.6 & 144.5 & 325.7 & 322.9 \\
\hline
\end{tabular}

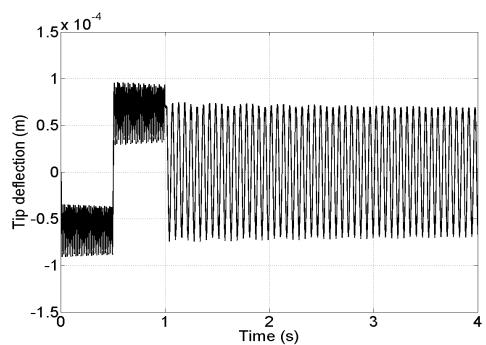

(a)

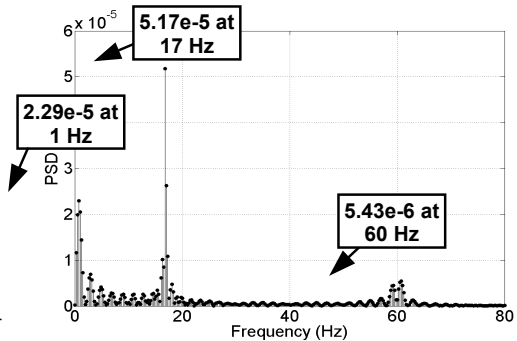

(b)

Figure 6: $\quad$ Case $1\left(\mathrm{~K}_{\mathrm{c}}=0\right)$ : Single-link flexible manipulator, (a) tip deflection w.r.t. shadow beam, and (b) FFT spectrum of tip deflection.

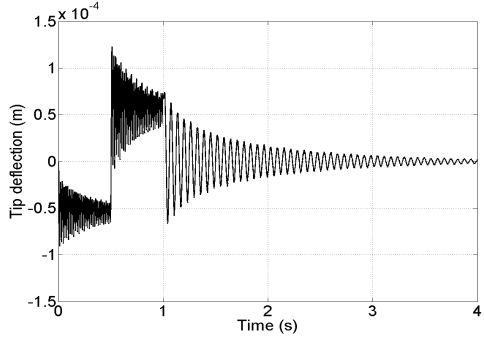

(a)

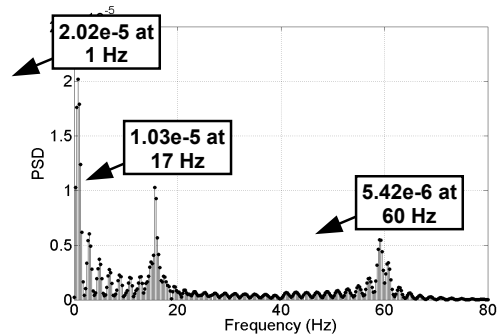

(b)

Figure 7: $\quad$ Case $3\left(\mathrm{~K}_{\mathrm{c}}=4.00 \mathrm{e} 5\right)$ : Single-link flexible manipulator. (a) tip deflection w.r.t. shadow beam, and (b) FFT spectrum of tip deflection. 
According to this figure, the peak values of the dominant frequencies, especially the main excitation frequency and the first natural frequency of the cantilever beam, were significantly reduced for Case 3 in comparison with Case 1 (Figure 6(b)). Three evaluation criteria, $E_{0-1}$ for $0 \leq t<1$ (during the manoeuvre), $E_{1-4}$ for $1 \leq t<4$ (at the end of the manoeuvre) and $E_{0-4}$ for $0 \leq t<4$ (the total response), were defined so as to compare the results. The values of these norms are the normalized tip deflections of the manipulator and were calculated using equations similar to those reported for cantilever beam (eqn 10). These norms are compared in Figure 8(a) for different gain values. As shown in this figure, the gain value for Case $3\left(K_{c}=4.00 e 5\right)$ had the smallest values of norms $E_{1-4}$ and $E_{0-4}$ in comparison with other gain values. Thus the vibration was more suppressed after the manipulator hub reached its the desired rotation $(1 \leq t<4)$ as well as the total response $(0 \leq t<4)$ for Case 3 . Another index which can be used to compare the results are values of the PSD peaks at the dominant frequencies. This index is shown in Figure 8(b) for different cases. Again for the three dominant frequencies, overall Case 3 shows the best result in suppressing the vibration.

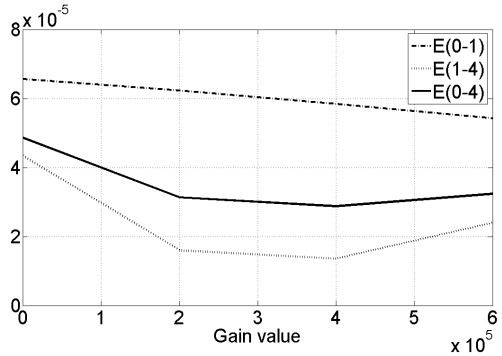

(a)

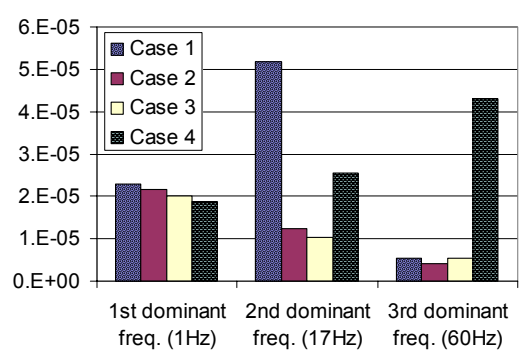

(b)

Figure 8: $\quad$ Single-link flexible manipulator, evaluation criteria for different gain values, (a) norms of vibration amplitudes, and (b) peak values of FFT results at dominant frequencies for different cases.

\section{Conclusions}

A piezoelectric actuator was successfully employed to suppress the vibration of a flexible link manipulator and the optimum values for the designed controller were found in this study. Finite element approach was used for modeling the cantilever beam and the robot arm. A linear position feedback controller was used in order to control the voltage applied to the piezoelectric actuator. The optimum location of the piezoelectric actuator was determined for the cantilever beam. It was shown that the arm vibration was well suppressed using the piezoelectric actuator. Also the frequency response of the tip deflection indicated that the dominant vibration frequencies corresponded well with those of the cantilever beam and rotating flexible manipulator. It was concluded that Case 3 
with a controller gain of $\mathrm{K}_{\mathrm{c}}=4.00 \mathrm{e} 5$ and the location of the piezoelectric actuator $30 \%$ of the beam length from the base produced the best results as far as suppressing the vibration was concerned.

\section{References}

[1] Lewis J. A., Inman D. J., Finite element modeling and active control of an inflated torus using piezoelectric devices. Journal of Intelligent Material Systems and Structures 2001; 12(12): 819-833.

[2] Khorrami F., Zeinoun I., Tome E., Experimental results on active control of flexible-link manipulators with embedded piezoceramics. Proceedings of IEEE International Conference on Robotics and Automation 1993; 3: 222-227.

[3] Chen C. Q., Shen Y., Optimal control of active structures with piezoelectric modal sensors and actuators. Smart Materials and Structures 1997; 6(4): 403-409.

[4] Peng F., Ng A., Hu Y., Actuator placement optimization and adaptive vibration control of plate smart structures. Journal of Intelligent Material Systems and Structures 2005; 16(3): 263-271.

[5] Moheimani, S. O. R., Ryall T., Considerations on placement of piezoceramic actuators that are used in structural vibration control. Proceedings of the 38th IEEE Conference on Decision and Control 1999; 2:1118-1123.

[6] Manning W. J., Plummer A. R., Levesley M. C., Vibration control of a flexible beam with integrated actuators and sensors. Smart Materials and Structures 2000; 9(6): 932-939.

[7] Kermani M. R., Patel R. V., Moallem M., Flexure control using piezostack actuators: design and implementation. IEEE/ASME Transactions on Mechatronics, 2005; 10(2): 181-188.

[8] Devasia S., Meressi T., Paden B., Bayo E., Piezoelectric actuator design for vibration suppression: placement and sizing. Journal of Guidance, Control, and Dynamics 1993; 16(5): 859-864.

[9] Bellezza F., Lanari L., Ulivi G., Exact modeling of the flexible slewing link. Proceedings of IEEE International Conference on Robotics and Automation 1990; 1: 734-739.

[10] De Luca A., Siciliano B., Closed-form dynamic model of planar multilink lightweight robots, IEEE Transactions on Systems, Man and Cybernetics, 1991, 21(4): 826-839.

[11] Gevarter W. B., Basic relations for control of flexible vehicles. AIAA Journal 1970; 8(4): 666-672.

[12] ANSYS Software ANSYS Inc., Canonsburg, PA, USA (www.ansys.com)

[13] Szyszkowski W., Youck D., Optimal control of a flexible manipulator. Computers and Structures 1993; 47(4): 801-813. 